\title{
Quit attempts and tobacco abstinence in primary care patients: follow-up of a pragmatic, two-arm cluster randomised controlled trial on brief stop-smoking advice $-A B C$ versus $5 A$ s
}

\author{
Sabrina Kastaun $\mathbb{1}^{1}$, Wolfgang Viechtbauer $\mathbb{C}^{2}$, Verena Leve ${ }^{1}$, Jaqueline Hildebrandt ${ }^{1}$, Christian Funke ${ }^{1}$, \\ Stephanie Klosterhalfen ${ }^{1}$, Diana Lubisch ${ }^{1}$, Olaf Reddemann ${ }^{1}$, Tobias Raupach $\mathbb{1}^{3,4}$, Stefan Wilm $\mathbb{( \mathbb { l }}^{1}$ and \\ Daniel Kotz $\mathbb{1}^{1,4,5}$
}

${ }^{1}$ Institute of General Practice (ifam), Centre for Health and Society (chs), Addiction Research and Clinical Epidemiology Unit, Medical Faculty of the Heinrich-Heine-University Düsseldorf, Düsseldorf, Germany. ${ }^{2}$ Dept of Psychiatry and Neuropsychology, Maastricht University, Maastricht, Netherlands. ${ }^{3}$ Institute for Medical Education, University Hospital Bonn, Bonn, Germany. ${ }^{4}$ Behavioral Science and Health, Institute of Epidemiology and Health Care, University College London, London, UK. ${ }^{5}$ Dept of Family Medicine, CAPHRI School for Public Health and Primary Care, Maastricht University, Maastricht, The Netherlands.

Corresponding author: Sabrina Kastaun (Sabrina.Kastaun@med.uni-duesseldorf.de)

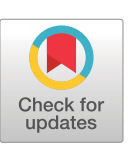

Copyright $\odot$ The authors 2021

This version is distributed under the terms of the Creative Commons Attribution NonCommercial Licence 4.0. For commercial reproduction rights and permissions contact permissions@ersnet.org

This article has supplementary material available from openres.ersjournals.com.

This article has been revised according to the correction published in ERJ Open Res 2021; 7: 50224-2021

[https://doi.org/10.1183/ 23120541.50224-2021]

Received: 31 March 2021

Accepted: 23 May 2021

First published: 26 July 2021

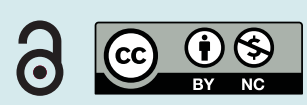

Shareable abstract (@ERSpublications)

GP-delivered brief stop-smoking advice increases smoking patients' quit attempts and abstinence rates. $3.5 \mathrm{~h}$ GP training in providing such advice according to the $A B C$ versus 5 As method increases advice rates. https://bit.ly/2SM3on4

Cite this article as: Kastaun S, Viechtbauer W, Leve V, et al. Quit attempts and tobacco abstinence in primary care patients: follow-up of a pragmatic, two-arm cluster randomised controlled trial on brief stop-smoking advice - ABC versus 5As. ERJ Open Res 2021; 7: 00224-2021 [DOI: 10.1183/ 23120541.00224-2021].

\section{Abstract}

We developed a 3.5-h training for general practitioners (GPs) in delivering brief stop-smoking advice according to different methods (ABC, 5As). In a pragmatic, cluster randomised controlled trial our training proved effective in increasing GP-delivered rates of such advice (from 13\% to 33\%). In this follow-up analysis we examined the effect of the training and compared $\mathrm{ABC}$ versus 5As on patient-reported quit attempts and point prevalence abstinence at weeks 4, 12 and 26 following GP consultation.

Follow-up data were collected in 1937 smoking patients - independently of the receipt of GP advice recruited before or after the training of 69 GPs. At week 26, 70\% of the patients were lost to follow-up. All 1937 patients were included in an intention-to-treat analysis; missing outcome data were imputed.

Quit attempts and abstinence rates did not differ significantly from pre- to post-training or between patients from the ABC versus the 5As group. However, ancillary analyses showed that patients who received GP advice compared to those who did not had two times higher odds of reporting a quit attempt at all followups and abstinence at week 26.

We reported that our training increases GP-delivered rates of stop-smoking advice, and the present analysis confirms that advice is associated with increased quit attempts and abstinence rates in patients. However, our training did not further improve these rates, which might be related to patients' loss to follow-up or to contextual factors, e.g. access to free evidence-based cessation treatment, which can hamper the transfer of GPs' advice into patients’ behaviour change.

Introduction

Owing to a lack of institutional, free stop-smoking services, the role of general practitioners (GPs) in Germany is of particular importance in the treatment of tobacco addiction. However, only around one fifth of smokers in Germany who consult a GP currently receive advice to quit smoking, and an even smaller minority receives a recommendation for adequate cessation treatment (e.g. nicotine replacement therapy (NRT), bupropion and varenicline) [1]. Evidence-based strategies are therefore needed to overcome barriers preventing GPs in Germany from routinely providing brief stop-smoking advice and recommendations for evidence-based cessation treatment to their smoking patients [2-5]. 
As such a strategy, we developed and pre-tested two 3.5-h trainings for GPs in delivering brief stop-smoking advice [6] aiming to address the most frequently mentioned barriers towards a routine provision of stop-smoking advice: the lack of training and skills of GPs on how to provide stop-smoking advice effectively and efficiently, and the lack of time during routine consultations [7-12]. One training was designed to instruct GPs how to deliver such advice according to the 5As counselling method (ask, advise, assess, assist, arrange, including the 5Rs in patients unmotivated to quit) [2], and the other to deliver the briefer ABC method (ask, brief advice, cessation support) [13].

In a previous pragmatic, two-arm cluster randomised controlled trial (cRCT), we showed that these trainings were highly effective at increasing the patient-reported rates of GP-delivered stop-smoking advice (primary outcome) and of delivered recommendations or prescriptions of behavioural and pharmacological smoking cessation treatment (secondary outcomes) [6, 14]. Furthermore, the vast majority of GPs experienced the training as useful for their daily practice ([14], supplementary data). A comparison of the effectiveness of the ABC versus the 5As method regarding these primary and secondary outcomes indicated that the ABC method may lead to higher rates of GP-delivered stop-smoking advice [14].

Evidence is strong that such advice significantly increases the rates of successful quitting, in particular when combined with pharmacological treatment $[15,16]$, and that training health professionals in advising their smoking patients can have measurable effects on continuous abstinence [17]. Hence, by increasing GP-delivered rates of advice on quitting and on effective treatment, we expected a higher number of smoking patients to intend to and to successfully quit following our GP trainings. However, among the few trials on the effectiveness of training particularly GPs [17-20] or GP trainees [12], so far only the studies of Verbiest et al. [21] and UnRoD et al. [20] examined the indirect effect of such GP training on the patients' cessation behaviour. Both studies found a positive effect of a 40-min individual training [20] or respectively a 1-h 5As group training [21] on GPs' counselling behaviour, but no [21] or, respectively, no clear [20] transfer of these effects on smokers' long-term quit rates, and no effects on smokers' attempts to quit $[20,21]$. Furthermore, studies conducted in the primary care context in Germany are lacking.

The present follow-up analysis of pre-specified [6], additional secondary outcomes of our above-mentioned cRCT therefore examined whether our 3.5-h training for GPs has the potential to indirectly increase patients' quit attempts and point prevalence abstinence rates at weeks 4, 12 and 26 following consultation with the trained GP. Furthermore, we compared the ABC and 5As training regarding these outcomes.

Methods

Study design and participants

The trial methods and primary outcome data are reported in detail elsewhere [6, 14]. Here, we briefly summarise the main methods with a focus on follow-up data assessment. Between June 2017 and February 2020 we conducted a pragmatic, two-arm cRCT training GPs in delivering brief stop-smoking advice according to two different methods of giving advice - ABC and 5As - with a pre-/post-design for the primary outcome as well as cluster randomisation for the comparison of both methods against each other. Ethics approval was obtained from the ethics committee at the Heinrich-Heine-University Düsseldorf, Germany (5999R), and all study participants provided written informed consent.

GP practices were recruited across the German federal state of North Rhine-Westphalia [6, 14]. A total of 52 GP practices (cluster) with 69 GPs were included in the statistical analyses of the primary and some secondary outcomes. Twenty-seven practices (32 GPs) were randomised (1:1) to receive training on the ABC method, and 25 practices (37 GPs) were randomised to receive training on the 5As [14].

Patients were eligible to participate if aged $\geqslant 18$ years, capable of providing informed consent, if they had an appointment with a study GP in person, and were current occasional or regular tobacco smokers (e.g. cigarettes, pipe, cigars, shisha). Patients who had participated in the pre-training data collection period were ineligible to participate in the post-training period.

\section{Procedures (intervention)}

In 2016, we had developed and pre-tested two 3.5-h trainings for GPs in delivering brief stop-smoking advice [6] according to either the 5As [2] or the briefer ABC [13] method. The development, based on the COM-B model of behaviour [22], and pilot testing have been described in the study protocol [6]. Both trainings included peer coaching (a senior researcher together with an experienced GP), lectures on tobacco addiction, on evidence-based cessation treatment, and the respective method of giving advice (ABC or 5As), followed by intensive, standardised role play with professional actors [6]. 
Assessment of primary and main secondary outcomes at baseline (main CRCT) [14]

Data on the primary outcome (rates of GP-delivered stop-smoking advice) and on main secondary outcomes (rates of GP-delivered recommendations of behavioural counselling, NRT, varenicline or bupropion) were collected during 6 weeks prior to and 6 weeks following the GP training in smoking patients consulting these GPs, by means of questionnaire-guided, face-to-face interviews conducted by members of the study team (baseline questionnaire: osf.io/7pmr5/, translated English version: osf.io/f2p7b/).

Assessment of secondary outcomes at follow-up: quit attempts, point prevalence abstinence

Patients who were smokers at baseline received a brief questionnaire by postal mail at 4, 12 and 26 weeks following their GP consultation, independently of whether they had received GP advice on quitting smoking at baseline (exemplary follow-up questionnaire at week 4: https://osf.io/u835j/, translated English version: https://osf.io/8uxag/). We pre-specified a time window for the postal dispatch of \pm 1 week for the first follow-up, and of \pm 2 weeks for the second and third follow-up. Patients who did not answer the first follow-up questionnaire received one reminder. We aimed to increase response rates by providing a small unconditional and non-financial incentive to the patients at each follow-up (e.g. a pen or some sweets).

The following secondary outcomes were collected at all three follow-up time points:

- Patient-reported rates of quit attempts since the baseline consultation with their GP.

- Patient-reported point prevalence abstinence rates since the baseline consultation with their GP, defined as the answer "I am still not smoking” on a question on the duration of their most recent quit attempt.

Although assessed, we decided not to analyse use of evidence-based stop-smoking treatments as a further secondary outcome, mainly because usage of such therapies is generally very low in Germany [23]. Assuming a decrease in response rates across all follow-up time points, the sample size was expected to be too small to draw reliable conclusions from such analyses. However, we report on the rates of usage of evidence-based pharmacological (NRT, varenicline, bupropion) or behavioural (single or group counselling, telephone counselling) treatment in patients who attempted to quit (supplementary table S1).

In order to attenuate recall bias and to avoid overlap with multiple GP consultations or quit attempts since the baseline consultation with their GP, all questions were thoroughly phrased with reference to the respective follow-up period.

\section{Statistical analysis}

Our study was powered to detect a clinically relevant effect of an increase in patient-reported rates of GP-delivered stop-smoking advice by at least $10 \%$ (OR 1.77) for the evaluation of the primary end-point $[6,14]$. We did not power the study for additional secondary outcomes or loss to follow-up.

\section{Analyses of the secondary outcomes collected at three follow-up time points}

Both the analysis plan (v3-3: osf.io/36kpc/) and statistical code (FU_rcode_v2-4: osf.io/5tfq7/) were written and published prior to the analyses. Writing of the code was based on a blinded dataset, i.e. with the values of the outcome variables in a randomly shuffled order. All analyses were conducted using $\mathrm{R}$ version 4.0.3 [24].

Data were structured hierarchically in clusters (= practices), with patients within these clusters. We used mixed-effects logistic regression models to analyse the secondary outcomes: self-reported quit attempts (yes/no) and self-reported point prevalence abstinence (yes/no) at all three follow-up time points. Models were adjusted for pre-specified potential confounders measured at baseline: patients' age, sex, level of education, and time spent with and strength of urges to smoke [25]. The group variable and its interaction with time (post- versus pre-training) were added to the models as fixed effects to compare the ABC versus the 5As method. Random intercepts and slopes (for the time effect) were included at the practice level. The time effect and the interaction were analysed by means of Wald-type tests (level of significance 0.05).

All 1937 patients who were smokers at baseline [14] were included in an intention-to-treat analysis. Multiple imputation was used to impute missing outcome data of each follow-up as well as missing data of potential confounders by chained equations ("mice-package" [26] with 20 imputed datasets and 10 iterations for each dataset). Results across the imputation datasets were pooled using Rubin's rules [27]. For plausibility checks, we looked at the summary statistics with minimum and maximum values, interquartile range, mean and median across all imputed datasets per outcome. We report ranges of

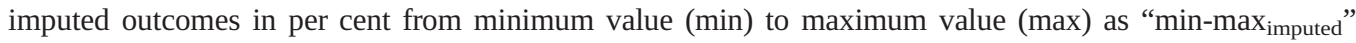
for the main descriptive results (observed percentages) presented within the Results section. 
Adherence to the protocol

All planned analyses are reported in the study protocol [6] and in the analysis plan (https://osf.io/36kpc/). In addition to the multilevel mixed-effects logistic regression models which we ran in $\mathrm{R}$ using the lme4 software package, we ran sensitivity analyses using two additional software packages (GLMMadaptive and glmmTMB) to increase confidence in the results.

As with the primary outcome analysis, we did not adjust the secondary outcome analyses as planned for motivation to stop smoking. Motivation was assessed directly following the consultation with the GP and might thus have been influenced by the GPs’ advice on smoking cessation.

We ran ancillary post hoc analyses exploring:

1) the effect of the receipt of stop-smoking advice (yes versus no) during the baseline consultation with the GP on the secondary outcomes at each follow-up, irrespective of the time variable (pre/post training) and the group variable (ABC or 5As training),

2) the effect of the receipt of stop-smoking advice (yes versus no) during the baseline consultation with the GP and its interaction with the time variable (post- versus pre-training) on the secondary outcomes at each follow-up, irrespective of group variable (ABC or 5As training) and

3) the effect of the receipt of a stop-smoking advice (yes versus no) during the baseline consultation with the GP and its interaction with the time variable (post- versus pre-training) and with the group variable (ABC versus 5As training) on the secondary outcomes at each follow-up.

Multilevel mixed-effects logistic regression models were also used for these analyses, including random intercepts and slopes (for receipt of advice and time; the latter only in the models for (2) and (3) at the practice level).

\section{Results}

The flow of study participants is depicted in a previous publication of this study [14]. In brief, 1937 smoking patients ( $n=1039$ were interviewed prior to and $n=898$ following the GP training) were eligible to receive follow-up questionnaires. Of these patients, 770 (39.8\%) answered the follow-up questionnaire at week 4, 616 (31.8\%) at week 12 and 558 (28.8\%) at week 26. Of these responders, around 2\% (1.7\% in week $4,1.9 \%$ in week 12 and $2.3 \%$ in week 26) had missing data on the outcome "quit attempt", and $\sim 5 \%$ (3.7\% in week 4, 7.1\% in week 12 and 3.4\% in week 26 ) of participants who had attempted to quit smoking did not provide information on their smoking status at follow-up.

Table 1 presents baseline sociodemographic and smoking characteristics of patients who participated in at least one of the three follow-up surveys (responders, $n=851,43.9 \%$ ) versus those who did not participate in any follow-up (non-responders, $n=1086,56.1 \%$ ). Responders appeared to be of older age, more likely to be female and reported the consumption of more cigarettes per day and higher urges to smoke than non-responders. Responders who reported a quit attempt had only very rarely used evidence-based pharmacological or behavioural cessation treatments to support this attempt (supplementary table S1).

\section{Self-reported quit attempts and point prevalence abstinence rates}

Rates of attempts to quit smoking since baseline were $18.9 \%$ (min-max imputed $_{16} 16.2 \%-20.2 \%$ ) at follow-up

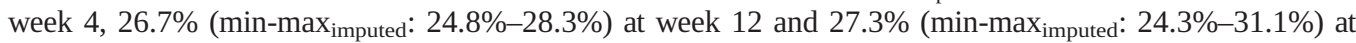
week 26. Table 2 shows that these rates did not differ significantly between the pre- and post-training group.

Point prevalence abstinence rates were $6.0 \%$ (min-max imputed : 4.4\%-8.3\%) at week $4, \quad 9.2 \%$

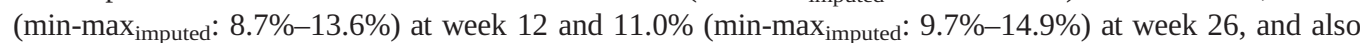
did not differ significantly between the pre- and post-training group (table 2).

Although the relative frequencies of reported quit attempts and point prevalence abstinence rates appeared to have increased more strongly from prior to following the training in patients whose GP had been trained according to the ABC method compared to the 5As method (adjusted ratio of the odds ratio (aROR) ranged from 1.07 and 1.71), no statistically significant interaction could be observed for both outcomes at any of the three follow-up time points (table 2).

\section{Ancillary analyses}

Results of the post hoc analyses 1) of the effect of the receipt of a stop-smoking advice (yes versus no) and 2) its interaction with the time variable (post- versus pre-training) are reported in table 3. Data showed that patients who received an advice to quit by their GP at the baseline consultation had around two times 


\begin{tabular}{|c|c|c|c|}
\hline & Responder & Non-responder & Total sample \\
\hline Subjects $n$ & 851 & 1086 & 1937 \\
\hline Age years (mean $\pm \mathrm{sD})$ & $50.9 \pm 14.9$ & $42.2 \pm 15.6$ & $46.1 \pm 15.9$ \\
\hline \multicolumn{4}{|l|}{ Sex } \\
\hline Female & $59.3(505)$ & 46.7 (507) & $52.3(1012)$ \\
\hline Male & $40.7(346)$ & $52.9(575)$ & $47.6(921)$ \\
\hline \multicolumn{4}{|l|}{ Level of education ${ }^{\#}$} \\
\hline High school equiv. & $22.1(188)$ & $22.2(241)$ & $22.2(429)$ \\
\hline Adv. techn. college equiv. & $12.9(110)$ & $15.0(163)$ & $14.1(273)$ \\
\hline Secondary school equiv. & $27.1(231)$ & $30.4(330)$ & $29.0(561)$ \\
\hline Junior high school equiv. & $36.1(307)$ & $27.7(300)$ & $31.3(607)$ \\
\hline No qualification & $1.8(15)$ & $4.6(50)$ & $3.4(65)$ \\
\hline Cigarettes/day (mean \pm sD) & $14.7 \pm 9.9$ & $13.8 \pm 9.6$ & $13.8 \pm 9.3$ \\
\hline Time spent with urges to smoke $[25]^{\natural}$ (mean \pm SD) & $3.1 \pm 1.5$ & $2.8 \pm 1.5$ & $2.9 \pm 1.5$ \\
\hline Strength of urges to smoke [25] (mean \pm SD) & $2.1 \pm 1.0$ & $2.0 \pm 0.9$ & $2.0 \pm 0.9$ \\
\hline Motivation to stop smoking [35] (mean \pm sD) ${ }^{+}$ & $3.4 \pm 1.8$ & $3.2 \pm 1.8$ & $3.3 \pm 1.8$ \\
\hline \multicolumn{4}{|c|}{$\begin{array}{l}\text { Data are presented as percentage (n), unless stated otherwise. Differences when calculating the tota } \\
\text { percentage can be explained by missing data on the respective variable. } \\
\text { "German equivalents to education levels listed in table from highest to lowest: high school equivalen } \\
\text { ("Allgemeine Hochschulreife"), advanced technical college equivalent ("Fachhochschulreife"), secondary schoo } \\
\text { equivalent ("Realschulabschluss"), junior high school equivalent ("Hauptschulabschluss") or no qualification. } \\
\text { "Both items of the Strength of Urges to Smoke Scale (SUTS) with values ranging from } 0=\text { lowest to } 6=\text { highes } \\
\text { urges. } \\
\text { "Items of the Motivation to Stop Smoking Scale ranging from } 1=\text { "I don't want to stop smoking" to } 7=\text { "I really } \\
\text { want to stop and intend to in the next month". }\end{array}$} \\
\hline
\end{tabular}

higher odds (adjusted odds ratio (aOR) between 1.90 and 2.01) of reporting a quit attempt at each of the three follow-up time points than patients who did not receive such an advice. Twenty-six weeks following baseline, patients who had been advised to quit smoking at baseline also had about two times higher odds of reporting abstinence from smoking than those who had not been advised to quit (aOR 2.22, 95\% CI $1.21-4.11)$.

Directions of the odds ratios suggest a larger increase of rates of quit attempts between pre- and post-training in patients who received advice to quit versus those who did not (aRORs between 1.27 and

TABLE 2 Patient reports on secondary study outcomes (attempt to quit smoking, point prevalence abstinence) at follow-up week 4, 12 and 26, stratified by pre/post data collection period and by training method of the GP they had consulted at baseline; and associations of these outcomes with training (post versus pre) and its interaction with the training method (ABC versus 5As by post versus pre)

\begin{tabular}{|c|c|c|c|c|c|c|c|c|}
\hline \multirow{2}{*}{$\begin{array}{l}\text { Outcome } \\
\text { (patient-reported) }\end{array}$} & \multicolumn{3}{|c|}{ Pre-training $\%$} & \multicolumn{3}{|c|}{ Post-training \% } & \multirow{2}{*}{$\begin{array}{l}\mathrm{aOR}_{\text {imputed }} \text { post versus } \\
\text { pre }(95 \% \mathrm{Cl})^{\#}\end{array}$} & \multirow{2}{*}{$\begin{array}{c}\text { aROR }_{\text {imputed }} A B C \text { versus } 5 \mathrm{As}^{\#} \text { by } \\
\text { post versus pre }(95 \% \mathrm{Cl})\end{array}$} \\
\hline & $\operatorname{Pre}_{A B C}$ & Pre $_{5 A s}$ & Pre $_{\text {total }}$ & Post $_{A B C}$ & Post $_{5 A s}$ & Post $_{\text {total }}$ & & \\
\hline Quit attempt at week 4 & 19.4 & 18.7 & 19.0 & 21.4 & 15.5 & 18.7 & $0.95(0.67-1.35)$ & $1.17(0.64-2.13)$ \\
\hline Quit attempt at week 12 & 25.8 & 28.1 & 26.9 & 30.6 & 21.6 & 26.5 & $1.02(0.72-1.45)$ & $1.12(0.65-1.93)$ \\
\hline Quit attempt at week 26 & 29.1 & 29.5 & 29.3 & 28.8 & 20.5 & 24.9 & $0.88(0.57-1.38)$ & $1.07(0.63-1.83)$ \\
\hline $\begin{array}{l}\text { Point prevalence abstinence } \\
\text { at week } 4\end{array}$ & 6.3 & 5.6 & 6.0 & 8.8 & 2.6 & 5.9 & $0.99(0.50-1.97)$ & $1.64(0.53-5.10)$ \\
\hline $\begin{array}{l}\text { Point prevalence abstinence } \\
\text { at week } 12\end{array}$ & 9.9 & 11.8 & 10.9 & 12.6 & 0.8 & 7.1 & $0.70(0.38-1.26)$ & $1.71(0.75-3.91)$ \\
\hline $\begin{array}{l}\text { Point prevalence abstinence } \\
\text { at week } 26\end{array}$ & 10.0 & 14.1 & 12.0 & 13.8 & 5.1 & 9.7 & $1.00(0.51-1.95)$ & $1.51(0.62-3.67)$ \\
\hline \multicolumn{9}{|c|}{$\begin{array}{l}\text { Data are presented as percentages (observed data), adjusted odds ratios (aOR), and adjusted ratios of the odds ratios (aROR) and 95\% confidence } \\
\text { interval ( } 95 \% \mathrm{CI} \text { ) around aOR and aROR (imputed data). } \\
\text { "Logistic regression models with a fixed effect for time (post- versus pre-training) and random intercepts and slopes (for the time effect) for the } \\
\text { practices; for the } \mathrm{ABC} \text { versus } 5 \mathrm{As} \text { comparison: the group variable (ABC versus } 5 \text { As training) and its interaction with time were added to the models } \\
\text { as fixed effects; both models were adjusted for patients' sex, age, level of education, time spent with urges to smoke and strength of urges to } \\
\text { smoke (Strength of Urges to Smoke Scale } 251 \text { ). }\end{array}$} \\
\hline
\end{tabular}


TABLE 3 Findings from ancillary analyses of patient reports on secondary study outcomes (attempt to quit smoking, point prevalence abstinence) at follow-up week 4, 12 and 26, stratified by whether the patient had received advice to quit smoking or not by the study GP they had consulted at baseline; and associations of these outcomes with the receipt of such advice (advice versus no advice) and its interaction with the time variable (advice versus no advice by post- versus pre-training)

\begin{tabular}{|c|c|c|c|c|c|c|c|c|}
\hline \multirow{2}{*}{$\begin{array}{l}\text { Outcome } \\
\text { (patient-reported) }\end{array}$} & \multirow{2}{*}{$\begin{array}{l}\text { No } \\
\text { advice } \\
\%\end{array}$} & \multirow{2}{*}{$\begin{array}{l}\text { Advice } \\
\%\end{array}$} & \multicolumn{2}{|c|}{ Pre-training } & \multicolumn{2}{|c|}{ Post-training } & \multirow{2}{*}{$\begin{array}{l}\text { aOR }_{\text {imputed advice }} \\
\text { versus no advice } \\
\quad(95 \% \mathrm{Cl})^{\#}\end{array}$} & \multirow{2}{*}{$\begin{array}{c}\text { aROR }_{\text {imputed }} \text { advice versus } \\
\text { no advice }{ }^{\#} \text { by post versus } \\
\text { pre }(95 \% \mathrm{Cl})\end{array}$} \\
\hline & & & $\begin{array}{l}\text { No } \\
\text { advice } \\
\%\end{array}$ & $\begin{array}{c}\text { Advice } \\
\%\end{array}$ & $\begin{array}{l}\text { No } \\
\text { advice } \\
\%\end{array}$ & $\begin{array}{c}\text { Advice } \\
\%\end{array}$ & & \\
\hline Quit attempt at week 4 & 16.6 & 26.6 & 18.6 & 21.7 & 13.4 & 29.2 & $1.90(1.28-2.82)$ & $1.27(0.64-2.53)$ \\
\hline Quit attempt at week 12 & 23.5 & 37.7 & 25.9 & 33.3 & 19.8 & 40.0 & $1.97(1.32-2.92)$ & $1.42(0.71-2.83)$ \\
\hline Quit attempt at week 26 & 24.1 & 38.8 & 28.3 & 35.7 & 17.6 & 40.5 & $2.01(1.43-2.82)$ & $1.34(0.71-2.53)$ \\
\hline $\begin{array}{l}\text { Point prevalence } \\
\text { abstinence at week } 4\end{array}$ & 5.7 & 6.9 & 6.1 & 5.0 & 5.0 & 7.8 & $1.49(0.65-3.39)$ & $0.95(0.27-3.35)$ \\
\hline $\begin{array}{l}\text { Point prevalence } \\
\text { abstinence at week } 12\end{array}$ & 8.9 & 10.4 & 10.6 & 12.8 & 6.2 & 9.1 & $1.21(0.63-2.33)$ & $1.13(0.40-3.19)$ \\
\hline $\begin{array}{l}\text { Point prevalence } \\
\text { abstinence at week } 26\end{array}$ & 9.4 & 16.7 & 10.9 & 19.0 & 7.1 & 15.4 & $2.22(1.20-4.11)$ & $1.04(0.43-2.52)$ \\
\hline
\end{tabular}

Data are presented as percentages (observed data), adjusted odds ratios (aOR), and adjusted ratios of the odds ratios (aROR) and 95\% confidence interval $(95 \% \mathrm{Cl}$ ) around aOR and aROR (imputed data). Odds ratios printed in bold are statistically significant $(p<0.05)$.

\# Logistic regression models with a fixed effect for advice (advice versus no advice) and random intercepts and slopes (for receipt of advice) for the practices; for the post- versus pre-training comparison: the time variable was added to the model as a fixed and random effect; both models were adjusted for patients' sex, age, level of education, time spent with urges to smoke and strength of urges to smoke (Strength of Urges to Smoke Scale [25]).

1.42; table 3). However, at none of the follow-ups were statistically significant interaction effects found neither for attempt to quit nor for tobacco abstinence (all $\mathrm{p}>0.05$ ).

Results of the post hoc analysis (3) on the effect of the receipt of a stop-smoking advice and its three-way interaction with the time variable and the group variable (advice versus no advice by post- versus pre-training by $\mathrm{ABC}$ versus 5As training) are not depicted in table 3. Neither of these interaction effects was statistically significant (all $\mathrm{p}>0.05$ ). Confidence intervals were extremely wide, making it difficult to draw reliable conclusions from these results.

\section{Discussion}

In this follow-up analysis of a cRCT, we examined the effect of a 3.5-h training for GPs in delivering brief advice to quit to their smoking patients and compared two methods of giving such advice - ABC versus $5 \mathrm{As}$ - on patients' self-reported smoking cessation behaviour - attempts to quit and point prevalence abstinence rates - at weeks 4, 12 and 26 following a routine consultation with the study GP. By means of post hoc analyses we further explored whether GPs' advice on quitting in this study was associated with quit attempts and success of these attempts in smoking patients at follow-up.

Whereas analyses of primary and secondary study outcomes (published elsewhere [14]) showed that our training substantially increased the rates of GP-delivered advice on quitting and recommendations of evidence-based cessation treatment, the present follow-up analysis did not confirm a transfer of this improved GP performance on patients' short- and long-term quitting behaviour. Although relative rates and direction of the ORs indicate a stronger increase of quit attempts and abstinence rates in patients whose GPs had been trained according to ABC compared to the 5As, neither attempts to quit nor abstinence rates differed significantly from pre- to post-training or in the ABC compared to the 5As group.

However, congruent with the existing evidence [15] a post hoc analysis demonstrated that smoking patients of our study who received GP advice on quitting smoking (irrespective of our training or of the ABC versus 5As comparison) compared to patients who did not receive such advice had two times higher odds of reporting a quit attempt at all follow-up time points and of reporting abstinence at week 26.

Hence, GPs' advice on quitting is effective in increasing patients' quit attempts and abstinence rates, and our training has proven effective in increasing these advice rates, but we still could not confirm a transfer of training effects measured at the GPs' level to patients' quitting behaviour. One can only speculate about the reasons for this paradox, but probable explanations might exist on different levels. First, the training 
provides content for GPs to advise smokers to quit but probably lacks in-depth content on how to assist smokers effectively with quitting, or because GPs cannot deliver the contents effectively to their patients. While the main results of the study [14] showed a significant increase from pre- to post-training in GP-delivered recommendation rates for evidence-based cessation treatment, the relative numbers of recommendations were still very low after the training $(<8 \%)$. Possibly, GPs may have reservations about the effectiveness and side-effects of stop-smoking medication. Such worries were in fact mentioned very often by the study GPs during the training and have previously been reported by other studies (e.g. Vogt et al. [28] and van Eerd et al. [29]). This might have not been adequately addressed by our brief training. However, physicians' advice to quit is most effective when combined with an offer of pharmacological or behavioural treatment [30].

Second, contextual factors might have hampered the transfer of GPs' effective advice and support into patients' behaviour change. For example, the very low usage rates of evidence-based smoking cessation treatment may be due to the lack of reimbursement of costs for such treatments in the German healthcare setting [23]. Evidence shows that the usage and free availability of such treatments substantially increase the rates of quit attempts and success [31]. Although more patients were advised to quit following the GP training, only those who could and were willing to afford effective treatment might have attempted to quit. Since we did not collect socioeconomic data of patients, we cannot verify this hypothesis, but recent population data from Germany showed that higher income is associated with higher odds of using medication to assist quit attempts [32].

In addition, limitations of the study design resulting in a small study sample at follow-up might have influenced the results measured at the patient level.

\section{Limitations}

First, around $70 \%$ of patients were lost to follow-up across the three follow-up time points, which might have affected the statistical power to detect group differences and reduce the generalisability of our results. However, by using multiple imputation methods and intention-to-treat analysis, we applied effective measures to reduce the potential for bias compared to a complete case analysis. Second, this is a secondary analysis. In a primary analysis, smoking patients would have had to be randomised into two groups (consultation following the GP training versus consultation prior to the GP training ("care as usual”)). Third, follow-up data were assessed by self-reports and tobacco abstinence lacked biochemical verification. Although, self-reported smoking status is a relatively reliable proxy for biomarker-validated smoking status $[33,34]$, data are susceptible to biases such as memory effects or social desirability bias, which might lead to an under- or over-reporting of the respective outcomes. A fourth limitation is that we were not able to analyse patient-reported use of evidence-based stop-smoking treatments as a secondary outcome of our GP training due to the very low usage rates of such treatments in Germany [23] (e.g. in our study, NRT was only used by around 3-15\% of smokers who attempt to quit) and due to the anticipated loss to follow-up. Such data would have provided a more detailed insight into the effectiveness of our training.

Further limitations which might have affected the results of the main cRCT also apply to the follow-up analysis including the fact that only short-term training effects on GPs' behaviour were studied, that we did not video record the baseline consultation and are thus not sure whether GPs had effectively implemented the $\mathrm{ABC}$ or $5 \mathrm{As}$ method, and that the study was not designed to compare the effectiveness of both training methods directly, which could only have been done with a non-pragmatic design [1].

The major strength is the pragmatic nature of this study, which was conducted in the real-world GP practice setting and during routine consultations, with outcome measures being assessed on both the GP and the patient level, the latter including long-term effects.

\section{Conclusions and policy implications}

Receiving brief stop-smoking advice during a routine consultation with a GP has a positive effect on patients' smoking cessation behaviour. However, our one-off 3.5-h training session for GPs in the provision of such advice according to the ABC versus the 5As method, which substantially increased advice rates on the GP level, could not further improve patients' attempts to quit smoking and long-term quit rates. Future training studies should more broadly take contextual factors into account which could hamper the transfer of GPs' effective advice and support into patients' smoking behaviour change - such as access to free evidence-based smoking cessation treatment. With regard to our training, for example, such a study should evaluate the additional effectiveness of financial incentive schemes for the reimbursement of treatment costs in smoking patients on their quit attempts and success. 
Author contributions: D. Kotz conceived the study and acquired funding for the current study together with S. Kastaun and V. Leve, and co-wrote this manuscript. S. Kastaun, V. Leve and D. Kotz developed and conducted the general practitioner training. S. Kastaun coordinated all study processes and wrote the first draft of the current manuscript. W. Viechtbauer, statistician, prepared the statistical analysis code, and advised on all statistical analyses together with D. Kotz and S. Kastaun, who conducted all analyses and interpreted the data. J. Hildebrandt, D. Lubisch, S. Klosterhalfen and C. Funke were mainly involved in all study processes including recruitment, data collection, data entry and cleaning. O. Reddemann, general practitioner and peer trainer, was mainly involved in the final evaluation of the training manual and the didactic methods. S. Wilm and T. Raupach gave valuable feedback at the time of designing the trial, and commented on and added to the present manuscript. All named authors contributed substantially to the manuscript and agreed on its final version. S. Kastaun and D. Kotz are the guarantors. The corresponding author attests that all listed authors meet authorship criteria and that no others meeting the criteria have been omitted.

This study is registered at www.drks.de with identifier number DRKS00012786. The data underlying this study are third-party data (de-identified participant data) and are available to researchers on reasonable request from the corresponding author (sabrina.kastaun@med.uni-duesseldorf.de). All proposals requesting data access will need to specify how it is planned to use the data and all proposals will need approval of the trial investigator team before data release. The study protocol, the statistical analysis plan (v3-3: https://osf.io/36kpc/) and statistical code (FU_rcode_v2-4: https://osf.io/5tfq7/) have been published.

Ethics approval: The study was approved by the medical ethics committee at the Heinrich-Heine-University Düsseldorf, Germany (5999R). All participants (patients and general practitioners) gave written informed consent prior to their inclusion in the study.

Conflict of interest: S. Kastaun has nothing to disclose. W. Viechtbauer has nothing to disclose. V. Leve has nothing to disclose. J. Hildebrandt has nothing to disclose. C. Funke has nothing to disclose. S. Klosterhalfen has nothing to disclose. D. Lubisch has nothing to disclose. O. Reddemann has nothing to disclose. T. Raupach reports personal fees from Pfizer, Novartis, GlaxoSmithKline, AstraZeneca and Roche as a speaker in activities related to continuing medical education, and grants from Pfizer and Johnson \& Johnson, outside the submitted work. S. Wilm has nothing to disclose. D. Kotz has nothing to disclose.

Support statement: The study was funded by the German Federal Ministry of Health (grant number ZMVI1-2516DSM221, Daniel Kotz). The funding sources had no role in the design and conduct of the study; collection, management, analysis, and interpretation of the data; preparation, review, or approval of the manuscript; or the decision to submit the manuscript for publication. Funding information for this article has been deposited with the Crossref Funder Registry.

\section{References}

1 Kastaun S, Kotz D. Brief medical advice on smoking cessation - results of the DEBRA study [Ärztliche Kurzberatung zur Tabakentwöhnung - Ergebnisse der DEBRA Studie]. J Addiction Res Pract 2019; 65: 34-41.

2 Fiore MC, Jaen CR, Baker TB, et al. A clinical practice guideline for treating tobacco use and dependence: 2008 update - a US public health service report. Am J Prev Med 2008; 35: 158-176.

3 National Institute for Clinical Excellence (NICE). Smoking: acute, maternity and mental health services, Guidance PH48. 2013. https://www.nice.org.uk/guidance/ph48 Date last accessed: 17 June 2020. Date last updated: March 2017.

4 Association of the Scientific Medical Societies (AWMF) [Arbeitsgemeinschaft der Wissenschaftlichen Medizinischen Fachgesellschaften (AWMF)]. S3 Guideline "Smoking and Tobacco Addiction: Screening, Diagnostics, and Treatment" [S3-Leitlinie "Rauchen und Tabakabhängigkeit: Screening, Diagnostik und Behandlung”]. AWMF-Register Nr. 076-006 2021. www.awmf.org/leitlinien/detail/ll/076-006.html Date last accessed: 1 February 2021.

5 Van Schayck OCP, Williams S, Barchilon V, et al. Treating tobacco dependence: guidance for primary care on life-saving interventions. Position statement of the IPCRG. NPJ Prim Care Respir 2017; 27: 38.

6 Kastaun S, Leve V, Hildebrandt J, et al. Effectiveness of training general practitioners to improve the implementation of brief stop-smoking advice in German primary care: study protocol of a pragmatic, 2-arm cluster randomised controlled trial (the ABCII trial). BMC Fam Prac 2019; 20: 107.

7 Strobel L, Schneider NK, Krampe H, et al. German medical students lack knowledge of how to treat smoking and problem drinking. Addiction 2012; 107: 1878-1882.

8 Twardella D, Brenner $\mathrm{H}$. Lack of training as a central barrier to the promotion of smoking cessation: a survey among general practitioners in Germany. Eur J Public Health 2005; 15: 140-145.

9 Hoch E, Franke A, Sonntag H, et al. Smoking cessation in primary medical care - chance or fiction? Results of the "Smoking and Nicotine Dependence Awareness and Screening (SNICAS)" study [Raucherentwöhnung in 
der primärärztlichen Versorgung - Chance oder Fiktion? Ergebnisse der "Smoking and Nicotine Dependence Awareness and Screening (SNICAS)"-Studie]. Addiction Med Res Practice 2004; 6: 47-51.

10 Raupach T, Merker J, Hasenfuss G, et al. Knowledge gaps about smoking cessation in hospitalized patients and their doctors. Eur J Prev Cardiol 2011; 18: 334-341.

11 Cancer Research UK. Smoking Cessation in Primary Care: A cross-sectional survey of primary care health practitioners in the UK and the use of Very Brief Advice. 2019. www.cancerresearchuk.org/sites/default/files/ tobacco_pc_report_to_publish___full12.pdf Date last accessed: 2 June 2020. Date last updated: March 2019.

12 Bobak A, Raupach T. Effect of a short smoking cessation training session on smoking cessation behaviour and its determinants among GP trainees in England. Nicotine Tob Res 2018; 20: 1525-1528.

13 Ministry of Health. The New Zealand Guidelines for Helping People to Stop Smoking. 2014. www.health.govt. nz/publication/new-zealand-guidelines-helping-people-stop-smoking Date last accessed: 6 June 2020.

14 Kastaun S, Leve V, Hildebrandt J, et al. Training general practitioners in the ABC versus 5 As method of delivering stop-smoking advice: a pragmatic, two-arm cluster randomised controlled trial. ERJ Open Res 2020; in press.

15 Stead LF, Buitrago D, Preciado N, et al. Physician advice for smoking cessation. Cochrane Database Syst Rev 2013; 5: CD000165.

16 Hartmann-Boyce J, Hong B, Livingstone-Banks J, et al. Additional behavioural support as an adjunct to pharmacotherapy for smoking cessation. Cochrane Database Syst Rev 2019; 6: CD009670.

17 Carson KV, Verbiest MEA, Crone MR, et al. Training health professionals in smoking cessation. Cochrane Database Syst Rev 2012; 5: CD000214.

18 Girvalaki C, Papadakis S, Vardavas C, et al. Training general practitioners in evidence-based tobacco treatment: an evaluation of the tobacco treatment training network in Crete (TiTAN-Crete) intervention. Health Educ Behav 2018; 45: 888-897.

19 McRobbie H, Hajek P, Feder G, et al. A cluster-randomised controlled trial of a brief training session to facilitate general practitioner referral to smoking cessation treatment. Tob Control 2008; 17: 173-176.

20 Unrod M, Smith M, Spring B, et al. Randomized controlled trial of a computer-based, tailored intervention to increase smoking cessation counseling by primary care physicians. J Gen Intern Med 2007; 22: 478-484.

21 Verbiest ME, Crone MR, Scharloo M, et al. One-hour training for general practitioners in reducing the implementation gap of smoking cessation care: a cluster-randomized controlled trial. Nicotine Tob Res 2014; 16: $1-10$.

22 Michie S, van Stralen MM, West R. The behaviour change wheel: a new method for characterising and designing behaviour change interventions. Implement Sci 2011; 6: 42.

23 Kotz D, Batra A, Kastaun S. Smoking cessation attempts and common strategies employed. Dtsch Arztebl Int 2020; 117: 7-13.

24 R Foundation for Statistical Computing. R: A language and environment for statistical computing. 4.0.3 version. Vienna, Austria, R Core Team, 2020.

25 Fidler JA, Shahab L, West R. Strength of urges to smoke as a measure of severity of cigarette dependence: comparison with the Fagerstrom Test for Nicotine Dependence and its components. Addiction 2011; 106 : 631-638.

26 van Buuren S, Groothuis-Oudshoorn K. Mice: multivariate imputation by chained equations in R. J Stat Softw 2011; 45: 1-67.

27 Rubin DB. Multiple Imputation for Nonresponse in Surveys. New York, John Wiley and Sons, 1987.

28 Vogt F, Hall S, Marteau TM. General practitioners' beliefs about effectiveness and intentions to prescribe smoking cessation medications: qualitative and quantitative studies. BMC Public Health 2006; 6: 277.

29 van Eerd EAM, Bech Risør M, Spigt M, et al. Why do physicians lack engagement with smoking cessation treatment in their COPD patients? A multinational qualitative study. NPJ Prim Care Respir 2017; $27: 41$.

30 Stead LF, Koilpillai P, Fanshawe TR, et al. Combined pharmacotherapy and behavioural interventions for smoking cessation. Cochrane Database Syst Rev 2016; 3: CD008286.

31 van den Brand FA, Nagelhout GE, Reda AA, et al. Healthcare financing systems for increasing the use of tobacco dependence treatment. Cochrane Database Syst Rev 2017; 9: CD004305.

32 Kastaun S, Brown J, Kotz D. Association between income and education with quit attempts, use of cessation aids, and short-term success in tobacco smokers: a social gradient analysis from a population-based cross-sectional household survey in Germany (DEBRA study). Addict Behav 2020; 111: 106553.

33 Patrick DL, Cheadle A, Thompson DC, et al. The validity of self-reported smoking: a review and meta-analysis. Am J Public Health 1994; 84: 1086-1093.

34 Vartiainen E, Seppälä T, Lillsunde $P$, et al. Validation of self reported smoking by serum cotinine measurement in a community-based study. J Epidemiol Community Health 2002; 56: 167-170.

35 Kotz D, Brown J, West R. Predictive validity of the Motivation To Stop Scale (MTSS): a single-item measure of motivation to stop smoking. Drug Alcohol Depend 2013; 128: 15-19. 